\title{
DOSIS ÓPTIMA PARA LA FERTILIZACIÓN NITROGENADA DEL ARROZ, EN LA REGIÓN CENTRAL DE PANAMÁ ${ }^{1}$
}

\author{
José Villarreal-Núñez ${ }^{2}$, Benjamín Name-Tuñón ${ }^{3}$, Jot Smyth $^{4}$, Erick Quirós-Valdés 5
}

\begin{abstract}
RESUMEN
Dosis óptima para la fertilización nitrogenada del arroz, en la Región Central de Panamá. El estudio se realizó en los años 2003 y 2004 en las Estaciones Experimentales de Calabacito y Guarumal y en fincas de productores de Mariato, Provincia de Veraguas y Tonosí, Provincia de Los Santos. Se aplicaron dosis de 0; 40; 80; 120; 160 y 200 $\mathrm{kg}$ de N/ha, con urea como fuente de N. Adicionalmente se aplicaron $100 \mathrm{~kg}$ de $\mathrm{P}_{2} \mathrm{O}_{5} /$ ha y $50 \mathrm{~kg}$ de $\mathrm{K}_{2} \mathrm{O} / \mathrm{ha}$, se empleó como fuentes superfosfato triple y cloruro de potasio, respectivamente. Se utilizaron las variedades IDIAP 2503 y CHI 3-30 en Calabacito y Guarumal; IDIAP 2503 y CR 5272 en Tonosí, Colombia XXI en Mariato. El diseño experimental fue de bloques completos al azar en un arreglo factorial con seis tratamientos y cuatro réplicas. Hubo respuesta significativa a la aplicación de $\mathrm{N}$ en todos los sitios. Los rendimientos obtenidos variaron desde 3,3 t/ha en Mariato a 5,7 t/ha en Tonosí, en el 2003. En Tonosí se obtuvieron dosis óptimas de $44 \mathrm{~kg} / \mathrm{ha}$ para IDIAP-2503 y $84 \mathrm{~kg} / \mathrm{ha}$ para CR5272. En Mariato $71 \mathrm{~kg} / \mathrm{ha}$ fue la dosis más efectiva para la variedad Colombia XXI. En 2004 en Calabacito se determinó que para CHI-330 no hubo respuesta a más de $55 \mathrm{~kg} / \mathrm{ha}$ aplicado y se estimó una eficiencia de uso de $\mathrm{N}$ de $35 \%$, sin embargo para IDIAP-2503 el nivel óptimo fue de $155 \mathrm{~kg}$ de $\mathrm{N} /$ ha y la eficiencia de utilización de $\mathrm{N}$ fue de $25 \%$. El nivel crítico foliar de $\mathrm{N}$ fue de 3,06\% para ambas variedades. En Guarumal se determinó que la dosis óptima de $\mathrm{N}$ para IDIAP-2503 fue de $119 \mathrm{~kg} / \mathrm{ha}$ y para CHI-330 de $105 \mathrm{~kg} / \mathrm{ha}$. El nivel crítico de $\mathrm{N}$ fue de 2,9 y 2,57, respectivamente.
\end{abstract}

Palabras clave: Arroz, dosis óptima de N, fertilización nitrogenada, nivel crítico, eficiencia de $\mathrm{N}$.

\begin{abstract}
Optimal rate of nitrogen fertilization for the rice, in the Central Region of Panama. The trials were conducted in 2003 and 2004 at the Calabacito and Guarumal Experiment Stations and on farms at Mariato, Veraguas and Tonosi, Los Santos. Nitrogen rates of 0, 40, 80, 120, 160 and $200 \mathrm{~kg} / \mathrm{ha}$ were applied as urea. All plots received 100 $\mathrm{kg} \mathrm{P}_{2} \mathrm{O}_{5} / \mathrm{ha}$ as triple superphosphate and $50 \mathrm{~kg} \mathrm{~K}_{2} \mathrm{O} / \mathrm{ha}$ as potassium chloride. Rice varieties IDIAP 2503 and $\mathrm{CHI}$ 0330 were compared at Calabacito and Guarumal; varieties IDIAP 2503 and CR 5272 were compared at Tonosi, and variety Colombia XXI was used at Mariato. A randomized complete block design with four replications was used in all experiments. There was a significant yield response to applied $\mathrm{N}$ in all experiments. Maximum grain yields in 2003 ranged from $3.3 \mathrm{t} / \mathrm{ha}$ at Mariato to $5.7 \mathrm{t} / \mathrm{ha}$ at Tonosi. Optimum N rates were $44 \mathrm{~kg} / \mathrm{ha}$ for IDIAP 2503 and $84 \mathrm{~kg}$ / ha for CR 5272, both at Tonosi, and $71 \mathrm{~kg} / \mathrm{ha}$ for Colombia XXI at Mariato. For the experiment at Calabacito in 2004, there was no yield response by variety CHI 0330 above $55 \mathrm{~kg}$ applied $\mathrm{N} /$ ha and fertilizer $\mathrm{N}$ use efficiency was estimated to be $35 \%$; optimum fertilizer N for IDIAP 2503 was $155 \mathrm{~kg} / \mathrm{ha}$ with a $25 \%$ use efficiency. Critical foliar $\mathrm{N}$ at flowering for both varieties at Calabacito was $3.06 \%$. Optimum fertilizer $\mathrm{N}$ levels at Guarumal were $119 \mathrm{~kg} / \mathrm{ha}$ for IDIAP 2503 and $105 \mathrm{~kg} / \mathrm{ha}$ for CHI 0330, with critical foliar N levels of 2.9 and $2.57 \%$, respectively.
\end{abstract}

Key words: Rice, optimal rate of $\mathrm{N}$, nitrogen fertilization, critical level, efficiency of $\mathrm{N}$.

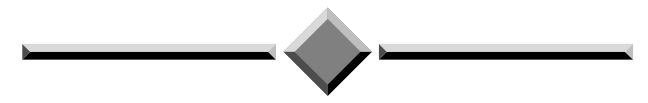

\footnotetext{
1 Recibido: 29 de junio, 2005. Aceptado: 5 de marzo, 2006. Trabajo presentado en la L1 Reunión Anual del PCCMCA. Panamá. Mayo, 2005.

2 Instituto de Investigación Agropecuaria de Panamá (IDIAP)-CIAC. Correo electrónico: jevilla38@gmail.com

3 Instituto de Investigación Agropecuaria de Panamá (IDIAP). Correo electrónico: bname63@gmail.com

4 Soil Science and plant nutrition. North Carolina State University. Raleigh, NC. USA. Correo electrónico: jot_smyth@ncsu.edu

5 Finca Experimental de Guarumal. IDIAP-CIAC. Correo electrónico: erick.quiros@idiap.gob.pa
} 


\section{INTRODUCCIÓN}

El uso de fertilizantes nitrogenados es considerado como esencial en la producción de arroz. La aplicación adecuada del nitrógeno $(\mathrm{N})$ depende del aporte del elemento suministrado por el suelo y de los requerimientos nutricionales del cultivo y sus variedades.

A través de muchos estudios se ha podido documentar la importancia de este elemento en la producción de granos (Raun y Johnson 1999; Ma et al.1999; Vetsch y Randall 2000).

El uso de este nutrimento puede aumentar sustancialmente los rendimientos del arroz, tomando en consideración que la eficiencia del uso del $\mathrm{N}$ varía con las características de la planta y las condiciones ambientales (Oaks 1994).

Históricamente el Laboratorio de Suelos del IDIAP, ha aceptado la teoría de la deficiencia de nitrógeno en los suelos tropicales y ha recomendado de forma general, según los resultados obtenidos en trabajos experimentales, niveles de aproximadamente 109 $\mathrm{kg} / \mathrm{ha}$. Actualmente se busca optimizar estas dosis de manera que la actividad sea económicamente rentable y ecológicamente sostenible. Por lo tanto, se requiere ajustar los requerimientos del $\mathrm{N}$, para recomendar dosis óptimas económicas a los productores.

En Panamá el arroz representa el alimento básico de la población y su cultivo es una importante fuente de empleo para el país. El excesivo uso de fertilización nitrogenada por parte de los productores no solo ha ocasionado desbalances nutricionales en el suelo, sino también, el peligro latente de contaminación de los acuíferos subterráneos.

El Nutrient Management Support System (NuMaSS), es un sistema experto en el manejo de nutrimentos que cuenta con una base de datos sobre el manejo de diversos cultivos en suelos tropicales de Asia, África y América Latina. Este programa permite obtener el diagnóstico de los posibles problemas de acidez o deficiencias que pueda presentar un suelo, realizar predicciones de las necesidades nutricionales de los cultivos y cálculos sobre los beneficios económicos de las prácticas agrícolas recomendadas.
La utilización del NuMaSS en las recomendaciones rutinarias del Laboratorio de Suelos permitirá mejorar la eficiencia de las mismas, una vez sea alimentado con datos reales provenientes de las investigaciones nacionales, que permitan cuantificar los nutrimentos requeridos por el cultivo y las mejores épocas para su aplicación.

Gran proporción del $\mathrm{N}$ utilizado por los productores de arroz en el país es aplicada a la superficie del suelo, al voleo. Esta forma de aplicación de fuentes de fertilizantes amoniacales permite la pérdida considerable de $\mathrm{N}$ por volatilización directa o por escorrentía, contribuyendo a la baja eficiencia de su uso y aumentando los peligros de contaminación de las fuentes de agua subterránea (Nyamangara et al. 2003; Dinnes y et al. 2002).

Trabajos realizados por Name y Villarreal (2004) en suelos ultisoles de Panamá, utilizando las variedades de arroz VIOAL-3189 e IDIAP L-7, demostraron que en este cultivo, la necesidad de $\mathrm{N}$ era mayor en las primeras etapas de crecimiento y disminuía a medida que la planta alcanzaba su madurez fisiológica. La variedad VIOAL-3189 logró una absorción máxima hasta de $89 \mathrm{~kg} / \mathrm{ha}$ mientras que la variedad IDIAP L-7 necesitaba un aporte de $112 \mathrm{~kg} / \mathrm{ha}$ hasta la etapa de maduración del grano. Esto muestra lo variable que son los requerimientos nutricionales del cultivo dependiendo de las características fisiológicas de cada variedad.

En zonas sometidas al cultivo continuo de arroz durante 14 años en Los Baños, Filipinas, se encontró que una absorción de $75 \mathrm{~kg}$ de $\mathrm{N}$ por hectárea incrementaba en $1,3 \mathrm{t} / \mathrm{ha}$ el rendimiento promedio del grano que fluctuaba entre 4,0 y 7,0 t/ha (Dobermann et al. 2000).

La forma de aplicación fraccionada del fertilizante permitió una mayor disponibilidad del $\mathrm{N}$ amoniacal para el cultivo, comprobado a través de un mayor rendimiento en materia seca y mayor contenido de $\mathrm{N}$ en la planta (Barrios y Adams 1992).

Una de las principales limitantes de este trabajo fue que en el segundo año no se pudo repetir el ensayo en todas las localidades programadas y además fue 
necesario realizarlo en un mayor número de localidades para una mejor recomendación en diferentes ambientes agroecológicos.

El objetivo primordial de realizar este ensayo fue determinar dosis óptimas de nitrógeno en el cultivo de arroz en suelos pertenecientes a importantes áreas arroceras del país, de tal forma que permita recomendar dosis óptimas económicas a los productores.

\section{MATERIALES Y MÉTODOS}

El ensayo se realizó durante el año 2003 en la Estación Experimental de Calabacito (IDIAP) y en fincas de productores del distrito de Mariato, provincia de Veraguas y Tonosí en la provincia de Los Santos y 2004 en las Estaciones Experimentales de Calabacito y Guarumal (IDIAP).

\section{Localización, suelos y clima}

La Estación Experimental de Calabacito se encuentra localizada a los $80^{\circ} 15^{\prime}$ de latitud norte y $81^{\circ} 5^{\prime}$ de longitud oeste, a una elevación de $100 \mathrm{msnm}$, con una precipitación y temperatura promedio de $2.500 \mathrm{~mm}$ y $27{ }^{\circ} \mathrm{C}$ respectivamente. Está ubicada en el bosque húmedo tropical (Holdridge 1967). El período de lluvia está comprendido entre los meses de mayo a diciembre. Políticamente se encuentra ubicada en el corregimiento de Remance, distrito de San Francisco, en la cuenca del Río Santa María.

El suelo es un Typic Plinthudult, fino, mezclado isohipertérmico, profundo, ácido, de estructura en bloques subangulares, baja capacidad de intercambio catiónico $\left(14 \mathrm{cmol}_{(+)} / \mathrm{kg}\right.$, promedio del perfil). El $\mathrm{pH}$ del perfil es ácido. El contenido de bases cambiables es muy bajo, ya que en el horizonte Ap ocupa sólo el 32\% de la CIC, mientras que en los horizontes más profundos oscila entre 1 y $6 \%$. En cuanto a las características mineralógicas en la fracción arcilla, domina la caolinita y aparecen en pequeñas cantidades vermiculita, goetita y gibsita. Esto explica la baja CIC del suelo y el estado avanzado de intemperismo que ha sufrido (Jaramillo 1991).

La Estación Experimental de Guarumal se encuentra ubicada al sur de la provincia de Veraguas, en la vertiente del pacífico sobre las coordenadas $7^{\circ} 46^{\prime} 50^{\prime}$ ' de latitud norte y $81^{\circ} 10^{\prime} 50$ " de longitud oeste, con una elevación de $10 \mathrm{msnm}$. Políticamente pertenece al corregimiento de Guarumal, distrito de Soná en la cuenca del río San Pablo. Los suelos representativos de la zona se clasifican como franco fino, mezclado, isohipertérmico ultic tropudalf. Se encuentran sobre sedimentos ígneos básicamente granodiorita (Jaramillo 1991). Pertenece según las zonas de vida de Holdridge (1967) al bosque húmedo tropical.

Mariato presenta suelos pertenecientes al orden inceptisol y se encuentra a unos $100 \mathrm{msnm}$, y presenta precipitación promedio anual de unos $3.000 \mathrm{~mm}$ de lluvia y temperatura promedio de $27^{\circ} \mathrm{C}$. Tonosí presenta suelos pertenecientes al orden vertisol y está a unos $45 \mathrm{msnm}$, con una temperatura promedio de $28^{\circ} \mathrm{C}$ y con una precipitación anual de $2.000 \mathrm{~mm}$. Ambas regiones según el diagrama para la clasificación de zonas de vida de Holdridge (1967) se ubican en el bosque tropical húmedo. El Cuadro 1 presenta las características físicas y químicas de los suelos de las fincas donde se plantó el ensayo.

Cuadro 1. Características físico-químicas del suelo de cada sitio experimental en las provincias de Veracruz y los Santos, Panamá. 2003.

\begin{tabular}{|c|c|c|c|c|c|c|c|c|c|c|c|c|c|}
\hline & A- $\underset{\%}{\text { L- Arc }}$ & $\begin{array}{c}\text { pH } \\
1: 2.5 \\
\text { agua- } \\
\text { suelo }\end{array}$ & $\begin{array}{l}\mathbf{P} \\
-\mathrm{mg} / \mathrm{l}\end{array}$ & $\begin{array}{l}\mathbf{K} \\
-\end{array}$ & $\begin{array}{l}\mathrm{Ca} \\
--\end{array}$ & $\begin{array}{r}\mathrm{Mg} \\
\mathrm{mol} / \mathrm{k}\end{array}$ & Al & $\begin{array}{c}\text { MO } \\
\%\end{array}$ & Mn & \multicolumn{4}{|c|}{$-----\mathrm{mg} / \mathrm{l}----\cdot$} \\
\hline Calabacito & $54-22-24$ & 5,1 & 1 & 82 & 0,54 & 0,15 & 3,2 & 2,16 & 211 & 235 & 5 & 5 & FARA \\
\hline Mariato & $36-44-20$ & 4,9 & 9 & 55 & 2,7 & 2,0 & 0,6 & 2,27 & 53 & 115 & 1 & 6 & $\mathrm{~F}$ \\
\hline Guarumal & $44-30-26$ & 4,8 & 8 & 31 & 10,0 & 3,2 & 0,4 & 2,41 & 41 & 96 & 3 & 6 & $\mathrm{~F}$ \\
\hline Tonosí & $36-30-34$ & 6,3 & 17 & 80 & 24,7 & 14,1 & 0,1 & 2,77 & 44 & 47 & 2 & 5 & FARC \\
\hline
\end{tabular}




\section{Tratamientos}

En las fincas de Calabacito, Guarumal y Tonosí se utilizaron seis tratamientos de nitrógeno $0 ; 40$; $80 ; 120 ; 160$ y $200 \mathrm{~kg} / \mathrm{ha}$. En Mariato fueron cinco los tratamientos de 0 a $160 \mathrm{~kg} / \mathrm{ha}$. Como fuente de $\mathrm{N}$ se aplicó urea al 46\%. En Calabacito, Guarumal y Tonosí, la aplicación de urea se fraccionó en tres aplicaciones: $1 / 3$ a la siembra, $1 / 3$ a los 30 días después de la siembra (dds) (máximo ahijamiento) y $1 / 3$ a los 60 dds (primordio floral). En Mariato, por ser suelos muy arenosos (84\%) la aplicación de $\mathrm{N}$ se fraccionó en cinco épocas diferentes: a la siembra, 30, 45, 60 y 75 dds. En este último suelo el $\mathrm{N}$ se pierde muy fácilmente por lixiviación disminuyendo la eficiencia de la fertilización.

En todos los casos se aplicó fertilización básica de $100 \mathrm{~kg}$ de $\mathrm{P}_{2} \mathrm{O}_{5} /$ ha, utilizando super fosfato triple ( 0-46-0) como fuente de fósforo y $50 \mathrm{~kg}$ de $\mathrm{K}_{2} \mathrm{O} / \mathrm{ha}$, empleando cloruro de potasio (0-0-60) como fuente de potasio. Se realizó el muestreo de suelo previo a la siembra y se recomendó la aplicación de cal en las fincas de Calabacito, Guarumal y Mariato.

Como material genético para realizar el ensayo se utilizó en Calabacito y Guarumal las variedades IDIAP- 2503 y CHI - 330. En Tonosí las variedades IDIAP - 2503 y CR - 5272 y en Mariato la variedad Colombia XXI.

Los tratamientos fueron dispuestos en el campo siguiendo un diseño experimental de bloques completos al azar en un arreglo factorial completo de seis tratamientos y cuatro réplicas. El área total del experimento fue de $270 \mathrm{~m}^{2}$. Cada parcela experimental presentaba un tamaño de cuatro metros de largo por dos de ancho, con una separación entre parcelas y entre repetición de $0,5 \mathrm{~m}$. La siembra se efectuó en forma manual a chorrillo a $0,20 \mathrm{~m}$ entre hileras a razón de $120 \mathrm{~kg}$ de semilla/ha.

Se midió el rendimiento total de grano, número de hijos en época de máximo ahijamiento, número de panículas por planta, peso de grano por panícula. Se realizó muestreo foliar a la floración del cultivo (hoja bandera) y muestra de follaje y grano a cosecha para determinar el contenido de nitrógeno total, según procedimiento empleado en el Laboratorio de Suelos del IDIAP (Villarreal y Name 1996). Además se midieron otras variables como vigor y altura de la planta, incidencia de Pyricularia grisea Sacc., número de panículas por tratamiento.

Los promedios de rendimiento de los tratamientos, contenido de nitrógeno en grano, en el follaje y en hoja bandera, se procesaron mediante análisis de varianza, por medio del paquete estadístico SAS (SAS System 1999).

Se determinó el nivel óptimo de N para cada variedad usando el modelo matemático de regresión Lineal - Plateau. Además se calculó el nivel crítico foliar de $\mathrm{N}$ para las variedades IDIAP-2503 y CHI-330 y la eficiencia de uso del fertilizante nitrogenado, calculada a través de la pendiente de la curva de regresión entre el $\mathrm{N}$ recuperado por el tratamiento (diferencia entre el $\mathrm{N}$ total absorbido menos el $\mathrm{N}$ absorbido por el tratamiento cero) y el $\mathrm{N}$ aplicado (no se calculó el nivel crítico para las variedades CR 5272 y Colombia XXI ya que no se pudo realizar el muestreo foliar).

\section{RESULTADOS Y DISCUSIÓN}

\section{Año 2003}

Hubo respuesta significativa a la aplicación de nitrógeno en todos los sitios donde se realizó el ensayo. En el año 2003 los rendimientos variaron desde 3,3 t/ha en Mariato hasta 5,7 t/ha en Tonosí. El rango óptimo de $\mathrm{N}$ varió desde $44 \mathrm{~kg} / \mathrm{ha}$ para la variedad IDIAP-2503 hasta $84 \mathrm{~kg} / \mathrm{ha}$ para la variedad CR-5272, ambas en Tonosí. En el suelo de Calabacito, se obtuvieron niveles óptimos de $73 \mathrm{~kg} / \mathrm{ha}$ para las variedades IDIAP-2503 y CHI-0330 (Figuras 1 y 2).

Para la variedad Colombia XXI en Mariato se determinó que $71 \mathrm{~kg}$ de N/ha era un nivel óptimo para este cultivar. Los bajos rendimientos fueron muy influenciados por prolongados períodos de sequía en etapas críticas del desarrollo del cultivo (Figura 1).

El modelo lineal - plateau utilizado, es muy práctico y exacto, explica por sí mismo que una vez 


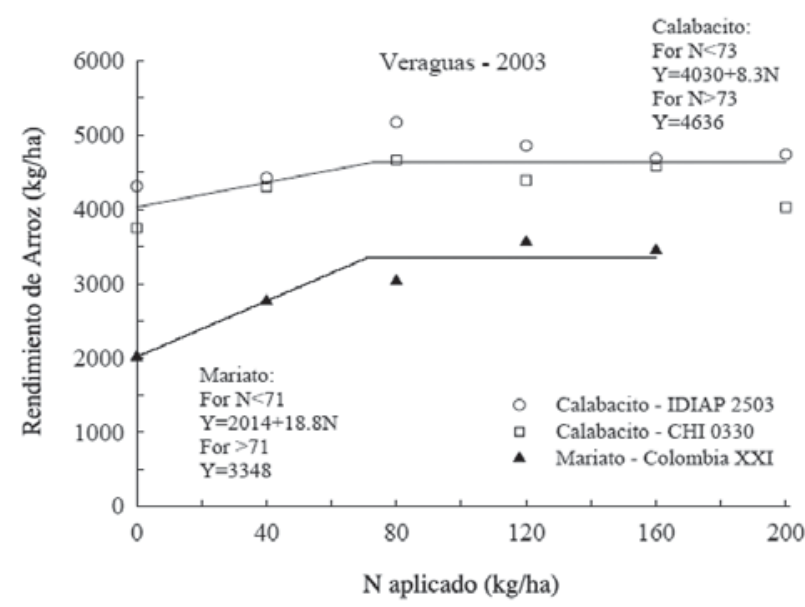

Figura 1. Respuesta de las variedades IDIAP 2503, CHI0330 y Colombia XXI a la aplicación de N. Estación Experimental Calabacito, Mariato, Veracruz, Panamá. 2003.

logrado el nivel óptimo de fertilización, aun cuando apliquemos cantidades mayores de fertilizante, el aumento en rendimiento no será tan significativo como para compensar las elevadas dosis.

La poca respuesta a la aplicación de $\mathrm{N}$ y el mayor rendimiento obtenido en el tratamiento al aplicar $0 \mathrm{~kg}$ de N/ha, en el suelo de Calabacito, estaba relacionado con el uso anterior de esta parcela, antes de la instalación de este ensayo. Este sitio fue utilizado durante varios años como banco de proteínas de forraje-leguminosa para el ganado. Esta respuesta limitada a la aplicación de $\mathrm{N}$ también fue confirmada a través de la determinación de la concentración de $\mathrm{N}$ en la hoja bandera en la etapa de floración. No se encontró diferencia significativa. El contenido del nutrimento en la hoja bandera se mantuvo elevado en todos los tratamientos. Además tampoco se observó deficiencias de ningún otro nutrimento (Cuadro 2). Debido al uso anterior que representa una limitante en esta parcela del ensayo, no se recomienda utilizar el resultado obtenido en esta parcela en suelos cuyo contenido de N sea menor.

Como se observa en el Cuadro 2, el tratamiento más cercano a la dosis óptima de $73 \mathrm{~kg} / \mathrm{ha}(80 \mathrm{~kg} / \mathrm{ha})$ es el que mostró el mayor contenido de $\mathrm{N}$ en la hoja bandera.

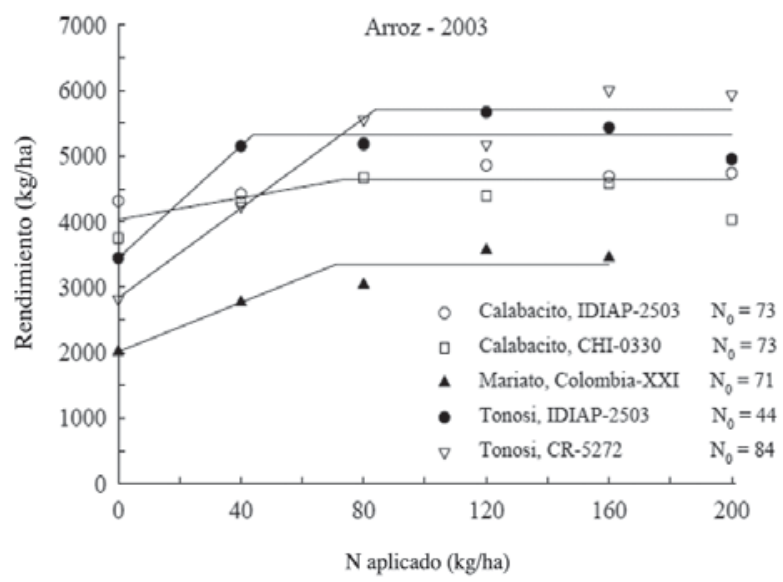

Figura 2. Nivel óptimo de aplicación de N para las variedades IDIAP-2503, CHI-0330, Colombia XXI y CR5272. Estación Experimental Calabacito, Mariato (Veracruz) y Tonosí (Los Santos), Panamá. 2003.

El Cuadro 3 presenta los resultados de algunas variables que fueron medidas en el ensayo como lo fueron: vigor y altura de la planta, incidencia de $P$. grisea Sacc., número de panículas por tratamiento, contenido de $\mathrm{N}$ en la hoja bandera, en el follaje y grano en época de cosecha, además del rendimiento.

Solamente se observó diferencia estadísticamente significativa en el contenido de $\mathrm{N}$ en el follaje (var. IDIAP-2503) y en grano (var. CHI-330), no así en el rendimiento ni en el número de panículas. En ambas variedades el rendimiento fue mayor en el tratamiento de $80 \mathrm{~kg}$ de $\mathrm{N} / \mathrm{ha}$, que concuerda con el resultado obtenido al realizar la regresión Lineal - Plateau que considera un nivel de $\mathrm{N}$ de $73 \mathrm{~kg} /$ ha como óptimo para ambas variedades.

Además se observó una baja incidencia en la afectación de $P$. grisea Sacc. y una altura normal de las plantas.

\section{Año 2004}

En el año 2004, el ensayo se repitió en las localidades de Calabacito y Guarumal utilizando las variedades 
Cuadro 2. Concentración de $\mathrm{N}$ y otros nutrimentos en hoja bandera en etapa de floración. Estación Experimental Calabacito (IDIAP), Panamá 2003.

\begin{tabular}{|c|c|c|c|c|c|c|c|c|c|c|}
\hline \multirow[t]{2}{*}{ Variedad } & \multirow{2}{*}{$\begin{array}{c}\text { N aplicado } \\
\text { kg/ha }\end{array}$} & $\mathbf{N}$ & $\mathbf{P}$ & $\mathbf{K}$ & $\mathrm{Ca}$ & Mg & Mn & $\mathbf{F e}$ & $\mathbf{Z n}$ & $\mathbf{C u}$ \\
\hline & & \multicolumn{5}{|c|}{$--------\%--------$} & \multicolumn{4}{|c|}{$------\mathrm{mg} / \mathrm{kg}------$} \\
\hline & 0 & 2,70 & 0,16 & 1,10 & 0,38 & 0,12 & 643 & 103 & 35 & 12 \\
\hline \multirow[t]{8}{*}{ IDIAP-2503 } & 40 & 2,85 & 0,16 & 1,07 & 0,40 & 0,13 & 745 & 110 & 45 & 12 \\
\hline & 80 & 3,01 & 0,19 & 1,16 & 0,41 & 0,14 & 685 & 110 & 33 & 13 \\
\hline & 120 & 2,86 & 0,16 & 1,06 & 0,43 & 0,14 & 745 & 113 & 53 & 13 \\
\hline & 160 & 2,57 & 0,18 & 1,06 & 0,37 & 0,13 & 625 & 110 & 35 & 13 \\
\hline & 200 & 3,00 & 0,18 & 1,14 & 0,36 & 0,13 & 510 & 135 & 35 & 13 \\
\hline & Promedio & 2,83 & 0,17 & 1,10 & 0,39 & 0,13 & 659 & 113 & 39 & 13 \\
\hline & DMS 0,05 & NS & NS & NS & NS & NS & NS & NS & NS & NS \\
\hline & 0 & 2,32 & 0,13 & 0,95 & 0,26 & 0,09 & 643 & 98 & 35 & 10 \\
\hline \multirow[t]{7}{*}{ CHI-0330 } & 40 & 2,18 & 0,14 & 0,94 & 0,29 & 0,11 & 483 & 85 & 38 & 10 \\
\hline & 80 & 2,44 & 0,14 & 0,97 & 0,28 & 0,11 & 408 & 70 & 33 & 10 \\
\hline & 120 & 2,20 & 0,16 & 0,93 & 0,28 & 0,11 & 495 & 73 & 40 & 10 \\
\hline & 160 & 2,43 & 0,14 & 0,91 & 0,30 & 0,11 & 438 & 95 & 38 & 10 \\
\hline & 200 & 1,86 & 0,17 & 1,08 & 0,26 & 0,11 & 330 & 75 & 35 & 10 \\
\hline & Promedio & 2,24 & 0,14 & 0,95 & 0,28 & 0,11 & 423 & 83 & 36 & 10 \\
\hline & DMS 0,05 & NS & NS & NS & NS & NS & NS & NS & NS & NS \\
\hline
\end{tabular}

Cuadro 3. Concentración de $\mathrm{N}$ en follaje y grano, vigor, altura de planta, incidencia de . grisea Sacc., número de panículas y rendimiento de grano en las var. IDIAP-2503 y CHI-330. Estación Experimental Calabacito (IDIAP), Panamá, 2003.

\begin{tabular}{|c|c|c|c|c|c|c|c|c|c|}
\hline \multirow[t]{3}{*}{ Variedad } & $\begin{array}{c}\mathbf{N} \\
\text { aplicado }\end{array}$ & Vigor & Altura & $\begin{array}{l}\text { P. orizae } \\
\text { Incidenc. }\end{array}$ & Panículas & $\begin{array}{c}\mathbf{N} \\
\text { Foliar }\end{array}$ & $\begin{array}{c}\mathbf{N} \\
\text { Follaje }\end{array}$ & $\begin{array}{c}\text { N } \\
\text { Grano }\end{array}$ & \multirow{2}{*}{$\begin{array}{c}\text { Rendimiento } \\
\text { kg/ha }\end{array}$} \\
\hline & \multicolumn{2}{|l|}{ kg/ha } & \multicolumn{2}{|c|}{$--\mathrm{cm}--$} & $\mathbf{n}^{\circ} / \mathbf{m}^{2}$ & \multicolumn{3}{|c|}{$-----\%----$} & \\
\hline & 0 & 5,5 & 71 & 1 & 63,3 & 2,70 & 0,73 & 1,20 & 4.311 \\
\hline \multirow[t]{8}{*}{ IDIAP-2503 } & 40 & 5,0 & 73 & 1 & 61,3 & 2,85 & 0,86 & 1,17 & 4.423 \\
\hline & 80 & 5,0 & 73 & 1 & 68,8 & 3,01 & 0,89 & 1,23 & 5.173 \\
\hline & 120 & 4,5 & 76 & 1 & 67,5 & 2,86 & 0,72 & 1,17 & 4.857 \\
\hline & 160 & 4,5 & 73 & 1 & 67,5 & 2,57 & 0,82 & 1,21 & 4.684 \\
\hline & 200 & 4,0 & 74 & 1 & 68,5 & 3,00 & 0,94 & 1,19 & 4.738 \\
\hline & Promedio & 4,8 & 73 & 1 & 66,1 & 2,83 & 0,83 & 1,19 & 4.698 \\
\hline & DMS 0,05 & NS & NS & NS & NS & NS & 0,15 & NS & NS \\
\hline & 0 & 6,0 & 68,0 & 3,5 & 67,0 & 2,32 & 0,79 & 1,16 & 3.748 \\
\hline \multirow[t]{7}{*}{ CHI-330 } & 40 & 5,0 & 71,0 & 2,8 & 78,8 & 2,18 & 0,88 & 1,11 & 4.302 \\
\hline & 80 & 5,5 & 71,0 & 3,5 & 67,8 & 2,44 & 0,89 & 1,25 & 4.669 \\
\hline & 120 & 5,5 & 71,0 & 4,8 & 65,3 & 2,20 & 0,80 & 1,17 & 4.394 \\
\hline & 160 & 5,0 & 72,0 & 3,5 & 80,5 & 2,43 & 0,94 & 1,27 & 4.585 \\
\hline & 200 & 6,0 & 71,0 & 4,8 & 75,8 & 1,86 & 0,94 & 1,35 & 4.029 \\
\hline & Promedio & 5,5 & 71,0 & 3,8 & 72,5 & 2,24 & 0,87 & 1,22 & 4.288 \\
\hline & DMS 0,05 & NS & NS & NS & NS & NS & NS & 0,14 & NS \\
\hline
\end{tabular}

P. orizae en una escala de $1-9$, siendo 9 muy alta. 
IDIAP-2503 y CHI-330. En 2004 por limitaciones logísticas y presupuestarias no se pudo continuar la investigación en Tonosí y Mariato, por lo tanto solo se utilizó el resultado de un año para recomendar la dosis óptima de N para CR 5272 y Colombia XXI.

\section{Calabacito}

En el Cuadro 4 se observa que hubo diferencias significativas al nivel de $5 \%$ para porcentaje de $\mathrm{N}$ en hoja bandera (floración), biomasa y granos en las dos variedades. También hubo diferencias significativas para $\mathrm{kg} / \mathrm{ha}$ de $\mathrm{N}$ absorbido por la biomasa, granos y el total absorbido por las dos variedades.

Por medio del cuadro anterior también podemos determinar el aporte de $\mathrm{N}$ proveniente del suelo de Calabacito. En el tratamiento de $0 \mathrm{~kg}$ de $\mathrm{N} /$ ha el aporte promedio de $\mathrm{N}$ del suelo fue de $57 \mathrm{~kg} / \mathrm{ha}$.

En Calabacito se encontró que para la variedad IDIAP-2503 el nivel óptimo para máximo rendimiento del cultivo fue lineal hasta $155 \mathrm{~kg}$ de $\mathrm{N}$ aplicados y para CHI-330 hasta los $55 \mathrm{~kg} /$ ha (Figura 3). La elevada respuesta de la variedad IDIAP-2503 confirma las observaciones hechas en el año 2003 sobre el gran aporte de $\mathrm{N}$ de parte del banco de proteínas que era mantenido en el sitio utilizado para el cultivo de esta variedad. También las condiciones climáticas,

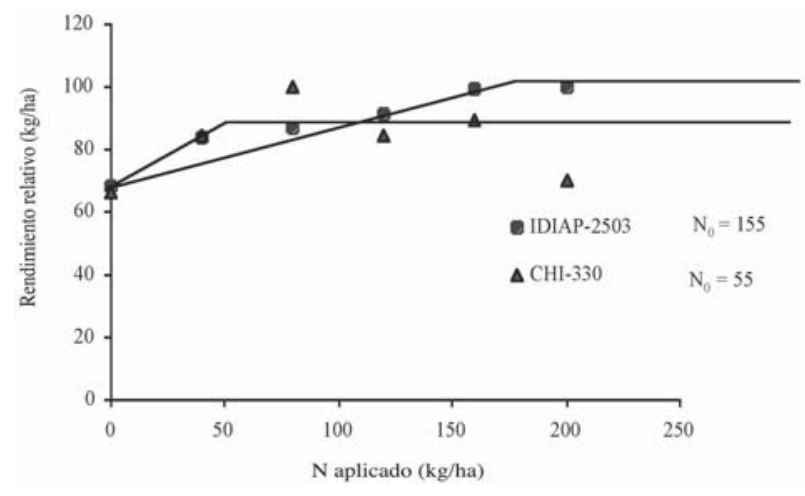

Figura 3. Respuesta de las variedades IDIAP 2503, CHI-0330 a la aplicación de N. Estación Experimental Calabacito (IDIAP), Panamá. 2004.

Cuadro 4. $\mathrm{N}$ absorbido por la hoja bandera y la biomasa, $\mathrm{N}$ absorbido por el grano y $\mathrm{N}$ total absorbido por el cultivo. Estación Experimental Calabacito (IDIAP), Panamá. 2004.

\begin{tabular}{|c|c|c|c|c|c|c|}
\hline Variedad & $\begin{array}{c}\mathrm{N} \\
\text { aplicado } \\
\text { kg/ha }\end{array}$ & $\begin{array}{c}\mathrm{N} \\
\text { foliar } \\
----\%\end{array}$ & $\begin{array}{c}\mathrm{N} \\
\text { biomasa } \\
\text { b-- - - }\end{array}$ & $\begin{array}{c}\mathbf{N} \\
\text { Abs. biomasa } \\
-----\end{array}$ & $\begin{array}{c}\mathrm{N} \\
\text { Abs grano } \\
--\mathrm{kg} / \mathrm{ha}--\end{array}$ & $\begin{array}{c}\mathrm{N} \text { total } \\
\text { Absorbido } \\
----\end{array}$ \\
\hline & 0 & 2,40 & 0,57 & 15,42 & 41,38 & 56,80 \\
\hline \multirow[t]{8}{*}{ IDIAP-2503 } & 40 & 2,56 & 0,77 & 29,28 & 50,96 & 80,23 \\
\hline & 80 & 2,74 & 0,78 & 28,73 & 52,99 & 81,72 \\
\hline & 120 & 2,77 & 0,82 & 29,34 & 55,45 & 84,79 \\
\hline & 160 & 3,03 & 0,95 & 36,40 & 60,49 & 96,89 \\
\hline & 200 & 3,18 & 1,12 & 40,60 & 60,96 & 101,57 \\
\hline & Promedio & 2,78 & 0,84 & 29,96 & 53,70 & 83,67 \\
\hline & DMS 0,05 & 0,17 & 0,20 & 14,86 & 11,48 & 23,33 \\
\hline & 0 & 2,60 & 0,83 & 21,69 & 36,41 & 58,10 \\
\hline \multirow[t]{7}{*}{ CHI-330 } & 40 & 2,72 & 0,80 & 25,32 & 46,64 & 71,95 \\
\hline & 80 & 2,86 & 0,86 & 31,43 & 55,18 & 86,61 \\
\hline & 120 & 2,54 & 0,96 & 29,04 & 46,52 & 75,57 \\
\hline & 160 & 2,35 & 1,09 & 24,95 & 49,31 & 74,26 \\
\hline & 200 & 2,38 & 1,19 & 36,71 & 38,45 & 75,16 \\
\hline & Promedio & 2,58 & 0,96 & 24,01 & 45,42 & 73,61 \\
\hline & DMS 0,05 & 0,19 & 0,17 & 9,47 & 10,62 & 14,79 \\
\hline
\end{tabular}


especialmente la variabilidad en el régimen pluviométrico, pudieron tener una gran influencia.

En la hoja bandera, durante la etapa de floración, se determinó el nivel crítico foliar para ambas variedades, encontrándose $3,06 \%$ de $\mathrm{N}$ como el nivel óptimo para condiciones de suelo y clima similares a las de Calabacito. Este valor se puede utilizar para el diagnóstico de posibles deficiencias del $\mathrm{N}$ en arroz (Figura 4).

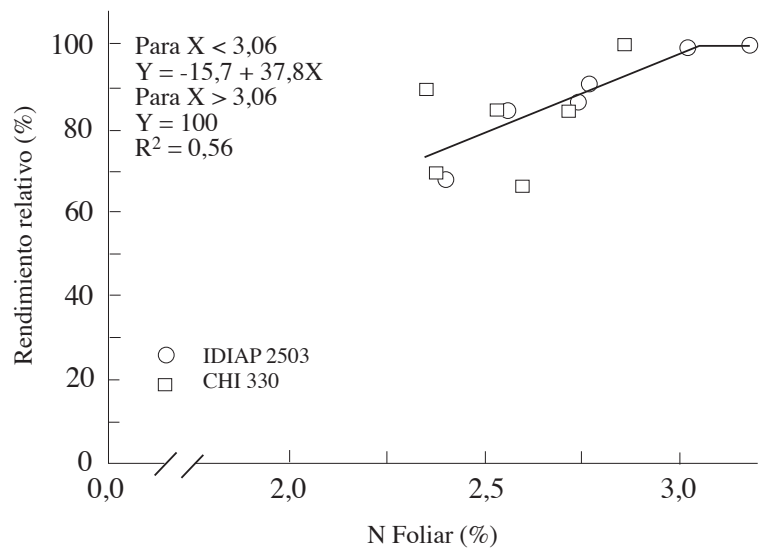

Figura 4. Nivel crítico de $\mathrm{N}$ en la biomasa de las variedades IDIAP-2503 y CHI-330. Estación Experimental Calabacito (IDIAP), Panamá. 2004.

Para CHI 330 no hay acumulación de N por más de $57 \mathrm{~kg}$ de N/ha aplicado. Es interesante recordar que el nivel óptimo para rendimiento de la misma variedad fue de $55 \mathrm{~kg}$ de N/ha. Para esta variedad la absorción fue lineal hasta $80 \mathrm{~kg}$ de N/ha. Para IDIAP-2503 la tendencia fue lineal hasta $200 \mathrm{~kg}$ de N/ha aplicados, o sea, para todos los niveles de $\mathrm{N}$ utilizados en el ensayo, esta variedad mostró respuesta a la absorción de $\mathrm{N}$ (Figura 5).

En la Figura 6 se puede ver que el $\mathrm{N}$ total absorbido por $\mathrm{kg}$ de rendimiento fue muy similar para las dos variedades - unos 20 gramos de $\mathrm{N}$ por cada $\mathrm{kg}$ de granos producido. Para ambas variedades podemos utilizar el nivel de $\mathrm{N}$ absorbido para predecir la producción de granos. La ecuación obtenida muestra un 82 de predicción.

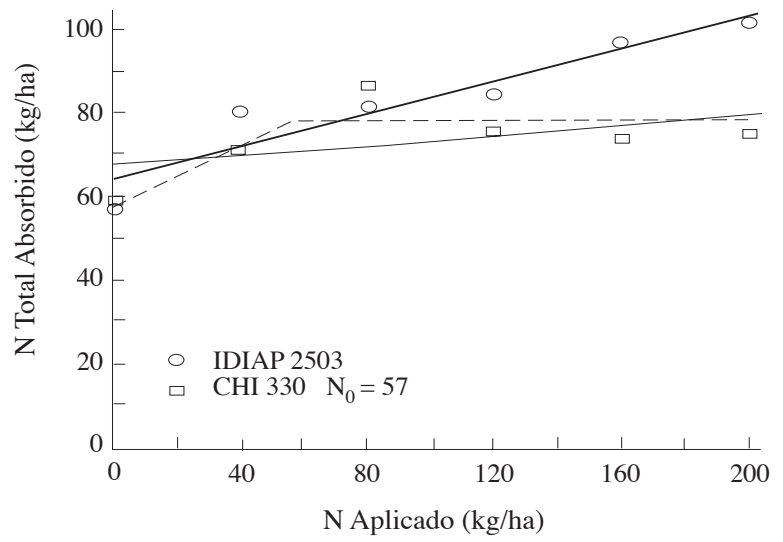

Figura 5. Absorción de $\mathrm{N}$ según dosis aplicada en las variedades IDIAP-2503 y CHI-330. Estación Experimental Calabacito (IDIAP), Panamá. 2004.

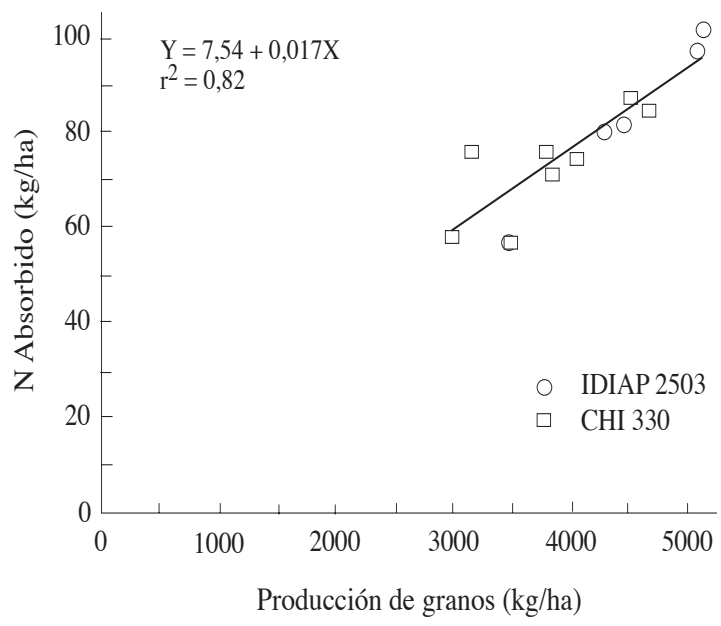

Figura 6. Nivel de absorción de $\mathrm{N}$ y producción de grano en las variedades IDIAP-2503 y CHI-330. Estación Experimental Calabacito (IDIAP), Panamá. 2004.

En la Figura 7 la línea de tendencia de la ecuación de regresión obtenida indica que la eficiencia de utilización del $\mathrm{N}$ aplicado con el fertilizante fue de $25 \%$ para la variedad IDIAP-2503, en el suelo de Calabacito. Con CHI 330 no hubo respuestas a más de $55 \mathrm{~kg}$ de N/ha; así la mejor estimativa de eficiencia de uso del $\mathrm{N}$ es el tratamiento con $40 \mathrm{~kg}$ de $\mathrm{N} / \mathrm{ha}$ aplicados, donde la eficiencia fue de $35 \%$. 


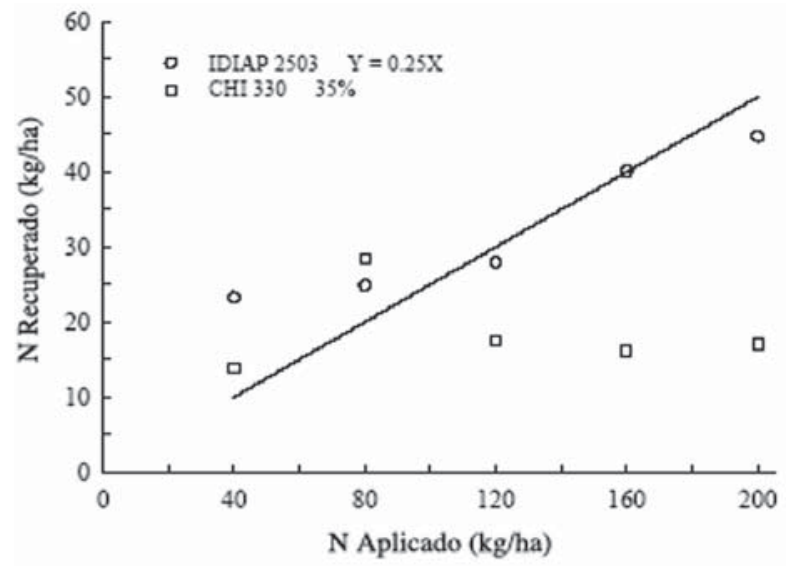

Figura 7. Eficiencia de utilización del $\mathrm{N}$ en las variedades IDIAP-2503 y CHI-330. Estación Experimental Calabacito (IDIAP), Panamá. 2004.

El $\mathrm{N}$ recuperado es la diferencia entre el $\mathrm{N}$ total absorbido por cada nivel de $\mathrm{N}$ aplicado y el $\mathrm{N}$ absorbido con el nivel de cero $\mathrm{kg}$ de $\mathrm{N} / \mathrm{ha}$. O sea, es el $\mathrm{N}$ absorbido que proviene del fertilizante. Así, la regresión para la variedad IDIAP-2503 fue forzada a pasar por la intersección, porque el $\mathrm{N}$ recuperado sin aplicar fertilizante debe ser cero $\mathrm{kg} / \mathrm{ha}$. Al determinar la pendiente de la regresión se obtuvo la eficiencia de utilización del $\mathrm{N}$ fertilizante por el arroz - $0,25 \mathrm{~kg}$ de N / kg de N aplicado, o sea 25\% para IDIAP-2503. Para el nivel de $80 \mathrm{~kg}$ de N/ha los valores de CHI-330 son muy similares a la regresión obtenida para la variedad IDIAP 2503.

\section{Guarumal}

En la localidad de Guarumal también se sembraron las variedades IDIAP-2503 y CHI-330. Esto sirvió para comprobar el efecto de las condiciones climáticas sobre la demanda de $\mathrm{N}$ del cultivo y el rendimiento. En Guarumal el régimen pluviométrico es mucho más estable que en Calabacito e incluso en años marcadamente secos como los años donde se siente el efecto del la corriente de El Niño, no se presenta un elevado déficit de agua para los cultivos (Name y Villarreal 2004).

En el Cuadro 5 se observan los resultados obtenidos para ambas variedades en los ensayos realizados en Guarumal. El contenido de $\mathrm{N}$ en la hoja bandera, en ambas variedades es menor que en Calabacito. Se observa también que hubo un gran porcentaje de granos vanos, sobre todo en la variedad IDIAP-2503, que mostró mayor susceptibilidad al ataque del ácaro Steneotarsonemus spinki,

Cuadro 5. Número de panículas, porcentaje de granos vanos, rendimiento de grano, producción de biomasa y concentración de $\mathrm{N}$ en la hoja bandera en las variedades IDIAP-2503 y CHI-330. Estación Experimental Guarumal (IDIAP), Panamá. 2004.

\begin{tabular}{|c|c|c|c|c|c|c|c|}
\hline \multirow[t]{2}{*}{ Variedad } & \multirow{2}{*}{$\begin{array}{c}\mathrm{N} \\
\text { aplicado } \\
\mathrm{kg} / \mathrm{ha}\end{array}$} & \multirow{2}{*}{$\begin{array}{c}\text { Número } \\
\text { de panículas } \\
\mathbf{n}^{\circ} / \mathbf{m}^{2}\end{array}$} & \multicolumn{2}{|c|}{ Número de granos } & \multicolumn{2}{|c|}{ Rendimiento } & \multirow{2}{*}{$\begin{array}{c}\text { N hoja } \\
\text { bandera } \\
\%\end{array}$} \\
\hline & & & $\begin{array}{c}\text { Total } \\
\mathrm{n} \% \mathbf{1 0} \text { panículas }\end{array}$ & $\underset{\%}{\operatorname{Vanos}}$ & $\begin{array}{l}\text { Biomasa } \\
----k g / h a\end{array}$ & $\begin{array}{l}\text { Granos } \\
----\end{array}$ & \\
\hline & 0 & 211 & 1.278 & 73 & 6.432 & 2.378 & 2,87 \\
\hline \multirow[t]{8}{*}{ IDIAP-2503 } & 40 & 208 & 1.160 & 67 & 6.442 & 2.885 & 2,87 \\
\hline & 80 & 190 & 1.430 & 75 & 7.520 & 2.995 & 2,95 \\
\hline & 120 & 223 & 1.335 & 63 & 7.577 & 3.568 & 2,92 \\
\hline & 160 & 240 & 1.236 & 60 & 8.092 & 3.281 & 3,16 \\
\hline & 200 & 225 & 1.172 & 72 & 7.927 & 3.172 & 3,14 \\
\hline & Promedio & 216 & 1.269 & 68 & 7.332 & 3.047 & 2,99 \\
\hline & DMS 0,05 & NS & NS & NS & NS & NS & NS \\
\hline & 0 & 207 & 1.118 & 42 & 4.806 & 2.678 & 2,52 \\
\hline \multirow[t]{7}{*}{ CHI-330 } & 40 & 254 & 1.333 & 43 & 6.316 & 3.460 & 2,55 \\
\hline & 80 & 231 & 1.395 & 43 & 7.178 & 3.585 & 2,76 \\
\hline & 120 & 202 & 1.244 & 42 & 6.384 & 4.035 & 2,73 \\
\hline & 160 & 260 & 1.278 & 43 & 6.889 & 3.912 & 3,05 \\
\hline & 200 & 250 & 1.375 & 53 & 9.862 & 3.977 & 3,00 \\
\hline & Promedio & 234 & 1.290 & 44 & 6.906 & 3.608 & 2,77 \\
\hline & DMS 0,05 & 40 & NS & NS & 2.690 & 861 & 0,34 \\
\hline
\end{tabular}


incluso en el tratamiento sin aplicación de $\mathrm{N}$, por lo que no resultó significativo la dosis de $\mathrm{N}$ aplicada sobre el porcentaje de granos vanos. Igualmente, a pesar de que hubo un aumento considerable en el rendimiento con el aumento de la dosis de $\mathrm{N}$ aplicada, éste no fue estadísticamente significativo para la variedad IDIAP-2503.

Para la variedad CHI-330 se obtuvieron diferencias estadísticamente significativas al nivel de $5 \%$ para el número de panículas producidas, rendimiento de grano y biomasa y contenido de $\mathrm{N}$ en la hoja bandera a floración.

La Figura 8 muestra que el nivel óptimo para la variedad IDIAP-2503 fue de $119 \mathrm{~kg}$ de N/ha y para CHI-330 fue de $105 \mathrm{~kg}$ de N/ha. Para ambas variedades utilizadas en el ensayo el nivel óptimo de $\mathrm{N}$ para máxima producción de grano fue mayor en Guarumal que en Calabacito. Los rendimientos variaron desde 3,6 t/ha para IDIAP-2503 hasta 4 t/ha para CHI-330. Los bajos rendimientos fueron consecuencia del severo daño causado por el ácaro S. spinki.

Se observó diferencia estadísticamente significativa en la absorción de $\mathrm{N}$ por la biomasa y el grano en

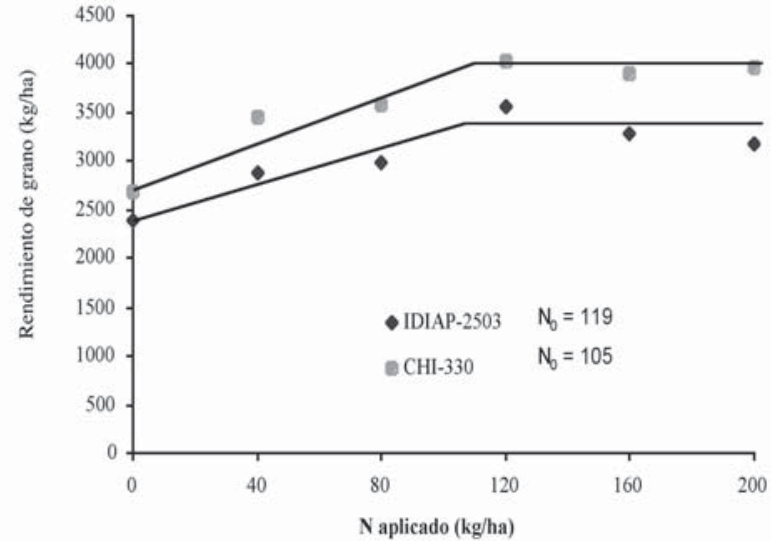

Figura 8. Nivel óptimo de N para las variedades IDIAP-2503 y CHI-330. Estación Experimental Guarumal (IDIAP), Panamá. 2004.

la variedad IDIAP-2503 y por la hoja bandera y el grano en CHI-330. Comparando con el ensayo realizado en Calabacito, hubo mayor absorción de $\mathrm{N}$ en Guarumal. Igualmente, en el tratamiento cero $\mathrm{kg}$ de $\mathrm{N} / \mathrm{ha}$, el aporte de $\mathrm{N}$ nativo del suelo de Guarumal fue mayor que el de Calabacito, siendo de $68 \mathrm{~kg} / \mathrm{ha}$ en IDIAP2503 y $58 \mathrm{~kg} / \mathrm{ha}$ para CHI-330 (Cuadro 6).

Cuadro 6. $\mathrm{N}$ absorbido por la hoja bandera, biomasa y grano, $\mathrm{N}$ total absorbido por la biomasa y el grano y $\mathrm{N}$ total absorbido por el cultivo. Estación Experimental Guarumal (IDIAP), Panamá. 2004.

\begin{tabular}{cccccccc}
\hline Variedad & $\begin{array}{c}\mathbf{N} \\
\text { aplicado }\end{array}$ & $\begin{array}{c}\mathbf{N} \\
\text { foliar }\end{array}$ & $\begin{array}{c}\mathbf{N} \\
\text { biomasa }\end{array}$ & $\begin{array}{c}\mathbf{N} \\
\text { grano }\end{array}$ & $\begin{array}{c}\mathbf{N} \\
\text { Abs. } \\
\text { Biomasa }\end{array}$ & $\begin{array}{c}\mathbf{N} \\
\text { Abs. } \\
\text { grano }\end{array}$ & $\begin{array}{c}\mathbf{N} \\
\text { Total } \\
\text { Absorb. }\end{array}$ \\
\hline kg/ha & $-----\%$ & ----- & $----1 /$ kga & ------- \\
\hline & 0 & 2,87 & 0,66 & 1,00 & 42,36 & 25,54 & 67,91 \\
& 40 & 2,87 & 0,81 & 1,26 & 53,66 & 36,24 & 89,90 \\
& 80 & 2,95 & 1,12 & 1,36 & 83,80 & 40,51 & 124,32 \\
& 120 & 2,92 & 1,03 & 1,49 & 77,92 & 52,76 & 130,68 \\
& 160 & 3,16 & 1,11 & 1,56 & 89,50 & 51,22 & 140,72 \\
& 200 & 3,14 & 1,08 & 1,59 & 84,99 & 50,33 & 135,02 \\
& Promedio & 2,99 & 0,97 & 1,38 & 72,04 & 42,77 & 114,75 \\
& DMS 0,05 & NS 330 & 0,23 & 0,12 & 22,61 & 10,54 & 24,62 \\
& 0 & 2,52 & 0,73 & 0,93 & 33,66 & 24,75 & 58,41 \\
& 40 & 2,55 & 0,65 & 1,06 & 41,13 & 36,35 & 77,48 \\
& 80 & 2,76 & 0,85 & 1,23 & 60,71 & 44,04 & 104,75 \\
& 120 & 2,73 & 0,83 & 1,34 & 50,24 & 54,08 & 104,32 \\
& 160 & 3,05 & 0,97 & 1,41 & 64,00 & 55,17 & 119,17 \\
& 200 & 3,00 & 0,94 & 1,46 & 92,66 & 58,08 & 150,74 \\
& Promedio & 2,77 & 0,83 & 1,24 & 57,07 & 45,41 & 102,48 \\
& DMS 0,05 & 0,34 & NS & 0,11 & 19,78 & 12,29 & 28,84 \\
\hline
\end{tabular}


En Guarumal se encontraron niveles críticos de $\mathrm{N}$ foliar diferentes para cada variedad. Para IDIAP-2503 fue de 2,9 y para CHI-330 se determinó que era de 2,57. Ambos fueron más bajos que los obtenidos con las mismas variedades en Calabacito (Figuras 9 y 10, respectivamente).

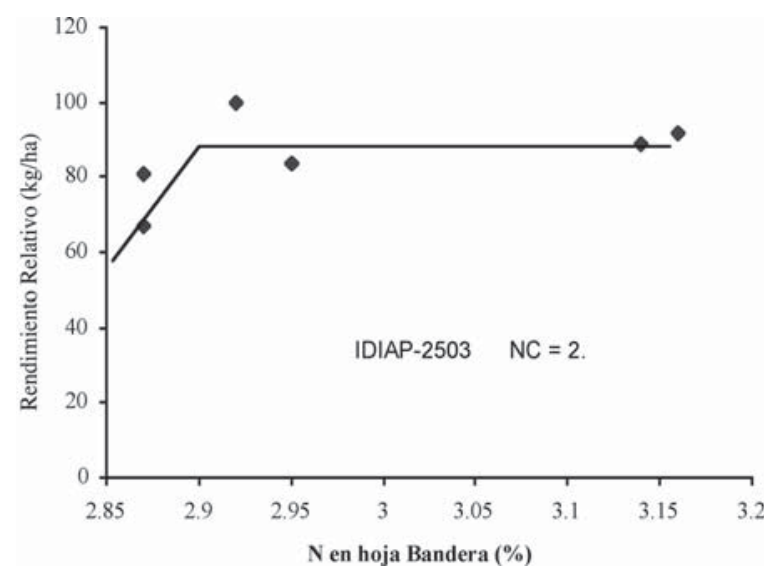

Figura 9. Nivel crítico foliar de $\mathrm{N}$ en la variedad IDIAP-2503. Estación Experimental Guarumal (IDIAP), Panamá. 2004.

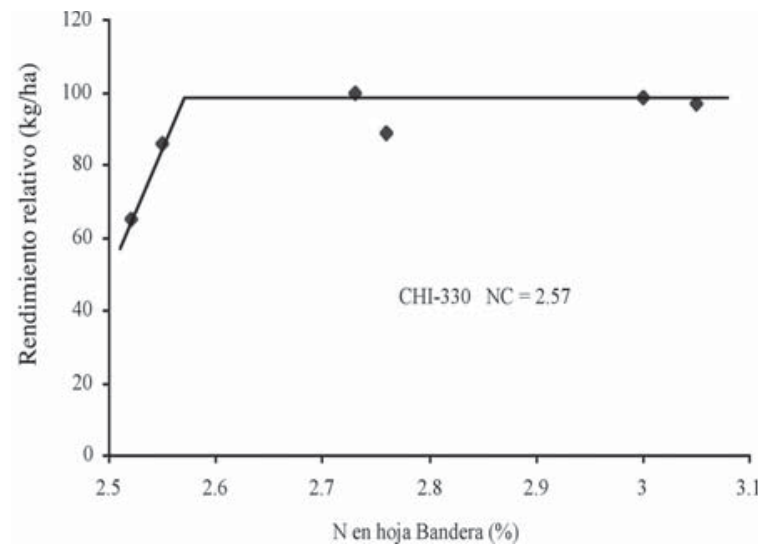

Figura 10. Nivel crítico foliar de $\mathrm{N}$ en la variedad CHI330. Estación Experimental Guarumal (IDIAP), Panamá. 2004.

El $\mathrm{N}$ total absorbido en Guarumal fue lineal hasta $94 \mathrm{~kg} /$ ha de N aplicado para CHI-330 y $99 \mathrm{~kg}$ de N/ha para IDIAP-2503. En Calabacito la absorción de esta última variedad era lineal hasta $200 \mathrm{~kg} / \mathrm{ha}$. Quizás el mayor suministro de $\mathrm{N}$ del suelo, una fertilización más fraccionada y un régimen de lluvias más homogéneo, contribuyó a la mayor absorción del N (Figura 11).

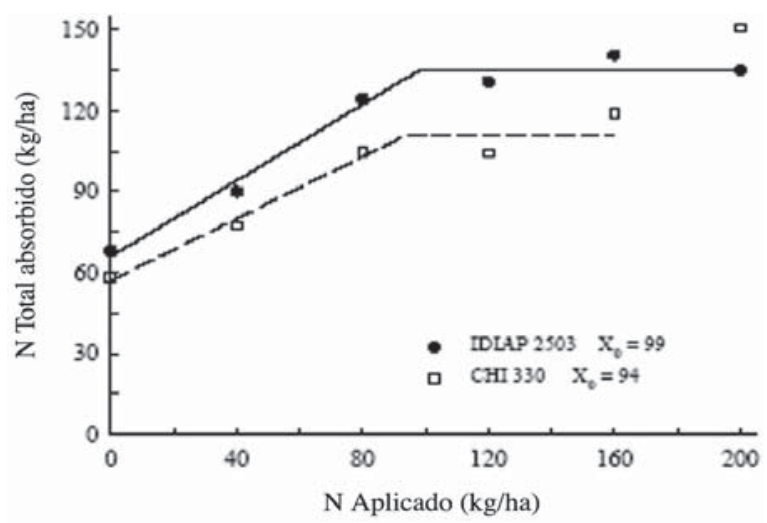

Figura 11. $\mathrm{N}$ total absorbido para las variedades IDIAP2503 y CHI-330. Estación Experimental Guarumal (IDIAP), Panamá. 2004.

También, la Figura 12 nos permite observar que la eficiencia de la aplicación de $\mathrm{N}$ en Guarumal, fue muy superior a la estimada en Calabacito. Para la variedad IDIAP-2503 se pudo recuperar hasta un $67 \%$ del N

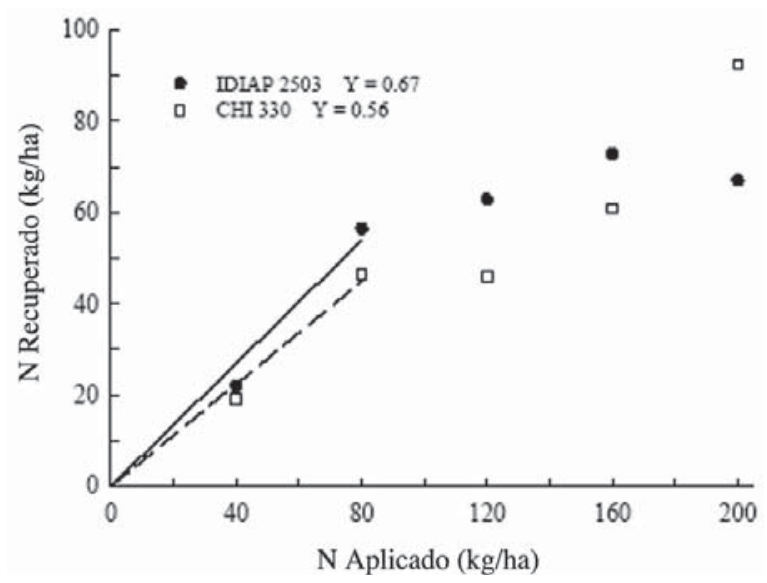

Figura 12. Eficiencia de recuperación del $\mathrm{N}$ en las variedades IDIAP-2503 y CHI-330. Estación Experimental Guarumal (IDIAP), Panamá. 2004. 
aplicado y $56 \%$ para CHI-330. Estos valores representan casi el doble de los obtenidos en Calabacito.

La relación entre $\mathrm{N}$ total absorbido y rendimiento de granos indica que por cada $\mathrm{kg}$ de grano producido el cultivo necesita absorber $0.06 \mathrm{~kg}$ de $\mathrm{N}$ total cuando se trata de la variedad IDIAP-2503 y $0,05 \mathrm{~kg}$ de $\mathrm{N}$ total absorbido en el caso de CHI-330 (Figura 13).

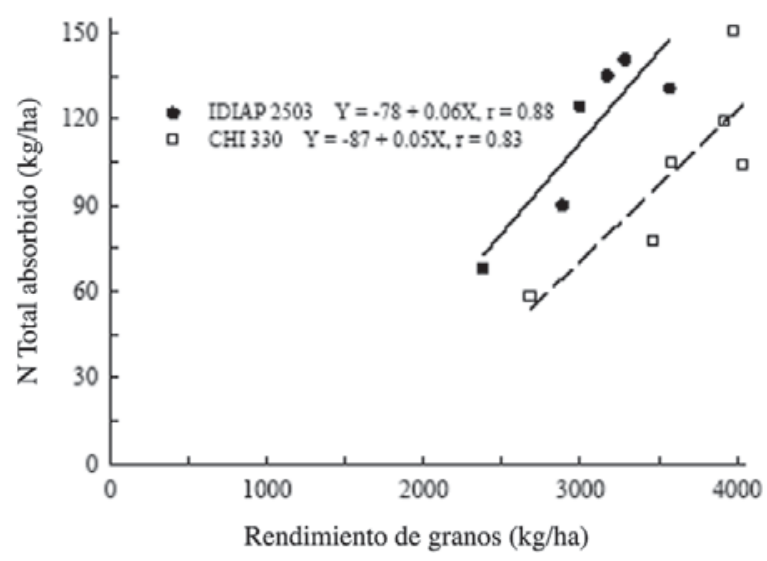

Figura 13. Nivel de absorción de $\mathrm{N}$ y producción de grano en las variedades IDIAP-2503 y CHI-330. Estación Experimental Guarumal (IDIAP), Panamá. 2004.

En Calabacito no se observó mucha diferencia entre variedades y el valor estimado fue menor - $0,02 \mathrm{~kg}$ de $\mathrm{N}$ absorbido -. Quizás esta diferencia entre sitios se deba a la mayor producción de biomasa por $\mathrm{kg}$ de rendimiento de grano en Guarumal comparado con Calabacito.

\section{CONCLUSIONES}

Hubo respuesta a la aplicación de nitrógeno en todos los sitios donde se desarrolló el ensayo, indicando que este es un factor limitante en los sistemas productivos.

Se encontró variabilidad en los rendimientos influenciado por las condiciones edafoclimáticas y la fisiología de las variedades utilizadas.
Se determinó que el suelo aporta una cantidad considerable de $\mathrm{N}$ al cultivo y aunque es necesario realizar aplicaciones del nutrimento en forma antropogénica, no es recomendable que éstas se hagan de manera excesiva (mayores de $110 \mathrm{~kg} / \mathrm{ha}$ ) que pueden traer como consecuencia desbalances nutricionales, suculencia de la planta y contaminación de acuíferos.

La determinación del nivel crítico de nitrógeno en la hoja bandera, en época de floración, permitirá detectar posibles deficiencias de este nutrimento en variedades nacionales a través del análisis de tejido vegetal.

La determinación de la cantidad de nitrógeno total absorbido, es una herramienta que permite estimar el rendimiento de grano del cultivo.

Los resultados encontrados permitirán mejorar las recomendaciones del Laboratorio de Suelos para el cultivo de arroz e incorporar datos de variedades nacionales en el programa experto NuMass, que permitan un uso mucho más racional del nitrógeno.

\section{LITERATURA CITADA}

BARRIOS, M.; ADAMS, M. 1992. Uso de fertilizantes nitrogenados en arroz irrigado en un suelo de la serie Calabozo. Revista Venezuelos, 4 (1 y 2): 28 - 35 .

DINNES, D.L.; KARLEN, D.L.; JAYNES, D.B.; KASPAR, T.C.; HATFIELD, J.L.; COLVIN, T.S.; CAMBARDELLA, C.A. 2002. Nitrogen management strategies to reduce nitrate leaching in tile-drained Midwestern soils. Agron. J. 94: 153 - 171.

DOBERMANN, A.; DAWE, D.; ROETTER, R.P.; CASSMAN, K.G. 2000. Reversal of rice yield decline in a long-term continuous cropping experiment. Agron. J. 92: $633-643$.

HOLDRIDGE, L.R. 1967. Life zone ecology. Tropical Science Center (Revised edition), San José, Costa Rica.

JARAMILLO, S. 1991. Pedones de campo y estaciones experimentales del IDIAP. Boletín Técnico $\mathrm{N}^{\circ} 38$. IDIAP. 
MA, B.L.; DWYER, L.M.; GREGORICH, E.G. 1999. Soil nitrogen amendment effects on nitrogen uptake and grain yield of maize. Agron. J. 91: 650 - 656.

NAME, B.; VILLARREAL, J. 2004. Dinámica de macro y micronutrimentos en arroz bajo riego en suelos ultisoles. In: Compendio de resultados de investigación del programa de suelos del IDIAP. Publicación conmemorativa del centenario de la República de Panamá. Instituto de Investigación Agropecuaria de Panamá. Panamá. 229 p.

NYAMANGARA, J. BERGSTROM, L. F.; PIHA, M.I.; GILLER, K.E. 2003. Use efficiency and nitrate leaching in a tropical sand soil, Journal of Environmental Quality 32: 599 - 606.
OAKS, A. 1994. Efficiency of nitrogen utilization in $\mathrm{C}_{3}$ and $\mathrm{C}_{4}$ cereals. Plant Physiol. 106: 407 - 414.

RAUN, W.R.; JOHNSON, G.V. 1999. Improving nitrogen use efficiency for cereal production. Agron. J. 91: $357-363$.

SAS SYSTEM. 1999. SAS Institute Inc. Versión 8. Cary, NC, USA.

VETSCH, J.A.; RANDALL, G.W. 2000. Enhancing no tillage systems for corn with starter fertilizers, row cleaners and nitrogen placement methods. Agron. J. 92: $309-331$.

VILLARREAL, J.E.; NAME, B. 1996. Técnicas analíticas del laboratorio de suelos del IDIAP. IDIAP. Divisa, Provincia de Herrera. 110 p. 